\title{
Diferencias de género en la toma de conciencia y vivencia de la propia orientación sexual
}

\author{
Juan Enrique Nebot-García \\ junebot@uji.es \\ Marta García-Barba \\ barbam@uji.es \\ Beatriz Gil-Juliá \\ bgil@uji.es \\ Cristina Giménez-García \\ giménezc@uji.es
}

\section{Resumen}

Introducción: La toma de conciencia y la aceptación de la propia orientación sexual pueden ser procesos largos y duraderos, sobre todo, en las personas con una orientación sexual no normativa. Variables como la presión sociocultural del entorno podrían favorecer que algunas personas experimenten dudas y malestar. Por este motivo, el presente trabajo tiene por objetivo analizar si existen diferencias de género en la toma de conciencia y vivencia de la orientación del deseo sexual.

Método: 100 personas que se definían como homosexuales cumplimentaron un cuestionario acerca de sus vivencias con la orientación sexual; con una media de edad de 24,55 años (DT $=4,15$ ), siendo el $50 \%$ hombres y el $50 \%$ mujeres.

Resultados: En relación con su propia orientación sexual, un $75 \%$ de jóvenes homosexuales ha tenido dudas y un $69 \%$ ha sentido malestar. En cuanto a la edad en la que definieron su orientación sexual, la media fue a los 14,69 años $(D T=3,83)$. En cuanto al género, solo se observan diferencias significativas en el malestar experimentado, siendo el $84 \%$ de los hombres, frente al $54 \%$ de mujeres, quienes sienten malestar debido a su orientación sexual $\left(X^{2}=10,51\right.$; $\mathrm{p}=0,001)$.

Conclusiones: Los resultados confirman una elevada prevalencia de malestar en población homosexual, que parece ser mayor en hombres. Estas diferencias en cuanto al género podrían guardar relación con los prejuicios que tiene la sociedad sobre la homosexualidad, siendo los hombres gays quienes más los sufren. Por tanto, sería conveniente que estas variables se tuvieran en cuenta a la hora de realizar estrategias de prevención.

Palabras clave: homosexualidad, orientación sexual, toma de conciencia, dudas, malestar. 


\begin{abstract}
Introduction: Awareness and acceptance of one's sexual orientation may be a long-lasting process, especially in people with a non-normative sexual orientation. Variables such as the pressure of the environment may promote that some people experience doubts and discomfort. Therefore, this study aims to analyze gender differences in the awareness and experience of sexual orientation. It would be necessary to examine if, as in past studies on psychosexual development, gender differences are also found in both variables.

Method: One hundred homosexual people completed a questionnaire about their experiences in sexual orientation. The average age was 24.55 years $(S D=4.15)$, being $50 \%$ men and $50 \%$ women.

Results: In relation to their own sexual orientation, $75 \%$ of young homosexuals have had doubts and $69 \%$ have felt discomfort. Regarding age of being aware about their sexual orientation, the average was 14.69 years $(S D=3.83)$. Regarding gender and sexual orientation, statistical significant differences were only observed in discomfort experienced, being $84 \%$ of men and $54 \%$ of women who feel it $\left(x^{2}=10.51, p=0.001\right)$.

Conclusions: The results confirm a high prevalence of discomfort among homosexual population, which seems to be higher in men. These gender differences may be related to society's prejudice about homosexuality, being gay men who suffer it the most. Therefore, these variables should be considered when prevention strategies are carried out.
\end{abstract}

Keywords: homosexuality, sexual orientation, awareness, doubts, discomfort.

\title{
Introducción
}

La conformación de la identidad psicosexual pasa por ser consciente y aceptar los propios deseos y sentimientos, al mismo tiempo que integrarlos dentro de una categoría de las posibles orientaciones sexuales y desarrollar un sentido de identidad con respecto a ella (Soriano 2004).

Una de las variables diferenciales en esta vivencia suele ser el contexto de socialización. Por ejemplo, las personas heterosexuales parecen ser menos conscientes de este proceso que coincide, en mayor medida, con los modelos sociales y los cánones esperados. En cambio, las personas homosexuales, al diferenciarse de la norma social, lo experimentan de manera más reflexiva. En línea con Soriano (2004), se vivenciaría un sentimiento de ser diferente al resto y, poco a poco, se iría tomando conciencia de los deseos homosexuales. Una vez la persona se autoidentifica como homosexual, empieza el proceso de aceptación y de integración de su homosexualidad dentro de la propia identidad.

Tal y como se ha comentado, todo este proceso puede llegar a ser un proceso difícil y complicado para las personas homosexuales, cuya identidad psicosexual debe estar, en muchos casos, escondida o asimilada a los patrones heteronomativos y machistas imperantes en la sociedad, influyendo negativamente en su estado de ánimo y su bienestar (Kosciw, Palmer y Kull 2015). Un claro ejemplo de ello es que en el mundo siguen existiendo 71 estados que criminalizan los actos sexuales entre personas adultas del mismo sexo en privado (Asociación Internacional de Lesbianas, Gays, Bisexuales, Trans e Intersex 2017). Otra consecuencia de esta presión social es que las propias personas homosexuales integran en su pensamiento muchos prejuicios 
sobre su orientación sexual creando, si cabe, más disonancia y mayor malestar. Esto es lo que se conoce como homofobia interiorizada (Newcomb y Mustanski 2010).

Por lo que respecta a España, durante los últimos años ha habido un gran progreso en cuanto a legislación a favor de los derechos de las personas LGTBI (lesbianas, gays, transexuales, bisexuales e intersexuales), como pueden ser la aprobación, en el 2005, del matrimonio de personas del mismo sexo y la posibilidad de adoptar (Ley 13/2005), el impulso de leyes a favor de la diversidad de género y los derechos de las personas transexuales que existen en algunas comunidades, como en la Comunidad Valenciana (Ley 8/2017) o la propuesta de ley contra la discriminación por orientación sexual, identidad o expresión de género y características sexuales y de igualdad social de lesbianas, gays, bisexuales, transexuales, transgénero e intersexuales (Boletín Oficial de las Cortes Generales núm. 122-1).

No obstante, pese a los importantes avances legislativos, en la sociedad no se observa tanta mejora. De hecho, las personas homosexuales aún siguen sufriendo discriminación, tanto en el ámbito social (Digoix et al. 2016) como en el ámbito escolar (Martxueta y Etxeberria 2014) y laboral (Digoix et al. 2016).

Esta influencia normativa parece repercutir de manera diferencial en hombres y mujeres. De hecho, los hombres homosexuales suelen sufrir mayor discriminación directa, siendo los que reciben más violencia física y verbal, en comparación con las mujeres (Nebot-García, Giménez-García, Ruiz-Palomino, Iglesias-Campos y BallesterArnal 2018), quizás porque los hombres, además de tener que enfrentarse a la heteronormatividad, tienen que hacer frente también al machismo de la sociedad, que castiga más los comportamientos homosexuales (Petersen y Hyde 2011; Poteat y Anderson 2012) y femeninos en los hombres (Carnaghi, Maass y Fasoli 2011; Glick, Gangl, Gibb, Klumpner y Weinberg 2007). En cambio, las mujeres parecen sufrir un tipo de discriminación menos directa, ya que se ha observado que tienen un menor acceso a los servicios médicos, en comparación con las mujeres heterosexuales (Agénor, Muzny, Schick, Austin y Potter 2017). Por otro lado, también existen diferencias en la edad en la que empiezan a realizarse las conductas homosexuales, siendo las mujeres las que lo hacen a una edad más tardía (Petersen y Hyde 2011).

Esta presión que ejerce la sociedad en la población homosexual, y sobre todo en la población masculina, puede influir en aspectos relevantes para la creación de su identidad psicosexual, como pueden ser la toma de conciencia y la aceptación de su propia orientación sexual, así como experimentar dudas o malestar con la propia orientación sexual (Soriano 2004). Por ello, el objetivo del presente trabajo es analizar si existen diferencias entre hombres y mujeres homosexuales respecto a las dudas con la propia orientación sexual, el malestar asociado y la edad en la que definieron su propia orientación sexual.

En consecuencia, se plantean las siguientes hipótesis: 1) mayor porcentaje de hombres habrá experimentado dudas con su orientación sexual, en comparación con las mujeres; 2) mayor porcentaje de hombres habrá experimentado malestar con su orientación sexual, en comparación con las mujeres; 3 ) las mujeres definirán su actual orientación sexual a una edad más tardía que los hombres. 


\section{Método}

\section{Participantes}

Del total de la muestra, se seleccionó al azar a un total de 100 personas que se identificaban como homosexuales, siendo el $50 \%$ hombres y el $50 \%$ mujeres. La edad promedio fue de 24,55 años (DT $=4,15)$. La mayoría de participantes tenían estudios universitarios (62\%) y eran ateos o agnósticos $(81 \%)$. Para poder formar parte de la investigación, los criterios de inclusión eran: tener entre 18 y 32 años y autoidentificarse con la orientación del deseo homosexual.

\section{Instrumentos}

Como no existía un cuestionario que se adaptara a nuestros objetivos, para esta investigación se creo un cuestionario ad hoc, en el que se evaluaba la vivencia sobre la propia orientación sexual. Además, también se preguntaron una serie de cuestiones sociodemográficas. Este instrumento consta de tres preguntas:

- Dudas con la propia orientación sexual. Esta escala está formada por un ítem en el que se pregunta "A lo largo de los años, ¿has dudado sobre tu orientación sexual?». Los participantes han de contestar siguiendo una escala tipo Likert $(0=$ Nada, $1=$ Algo, $2=$ Bastante, $3=$ Mucho $)$. Para facilitar la interpretación de los resultados, las respuestas de los ítems fueron recodificadas en dos grupos. Por una parte, están las personas que no tenían ningun tipo de duda («Nada») y, por otra, aquellas que mostraban algún grado de duda («Algo», «Bastante» o «Mucho»).

- Malestar con la propia orientación sexual. Esta escala consta de un ítem en el que se pregunta «A lo largo de los años, ¿cuánto malestar has sentido sobre tu orientación sexual?». Los participantes deben responder siguiendo una escala tipo Likert ( $0=$ Nada, $1=$ Algo, $2=$ Bastante, $3=$ Mucho $)$. Del mismo modo que con el ítem anterior, se recodificaron las respuestas en dos grupos. Por una parte, se agruparon las personas sin malestar («Nada») y, por otra, aquellas que referenciaban haber sentido algún grado de malestar («Algo», «Bastante» o «Mucho»).

- Edad en la que se definió su actual orientación sexual. En este ítem se pregunta "¿A qué edad definiste tu actual orientación sexual?» y debe responderse indicando un número.

\section{Procedimiento}

Para la recogida de participantes, la información sobre el estudio se difundió a través de las redes sociales, grupos de interés e informantes clave. Desde sus dispositivos móviles, las personas interesadas podían acceder al formulario online, donde se les informaba del carácter anónimo, voluntario y confidencial de la investigación y se les pedía el consentimiento informado. Una vez dado dicho consentimiento, podían acceder al cuestionario para cumplimentarlo. De entre las personas que participaron, se seleccionó al azar un grupo de hombres y mujeres que se identificaban como homosexuales. 


\section{Analisis estadísticos}

Para realizar los diferentes análisis, se utilizó el programa estadístico SPSS 22. En primer lugar se realizaron análisis de frecuencias y descriptivos para obtener los datos demográficos de las personas participantes. Asimismo, para analizar las diferencias de género en las dudas y el malestar con la propia orientación sexual, se realizaron tablas de contingencias y pruebas de chi cuadrado. $\mathrm{Y}$, por último, para calcular las diferencias de género en la edad de toma de conciencia de la orientación sexual, se realizó una prueba t de Student.

\section{Resultados}

En términos generales, se observa que, en relación con su propia orientación sexual, un $75 \%$ de jóvenes homosexuales ha tenido dudas y un $69 \%$ ha sentido malestar. Si se separan estos datos entre hombres y mujeres, se observa que, en comparación con las mujeres, los hombres muestran más dudas y mayor malestar con su orientación sexual (véase la figura 1). No obstante, las diferencias en las dudas no fueron significativas $\left(X^{2}=0,48 ; p=0,488\right)$; en cambio, en el malestar experimentado, sí $\left(x^{2}=10,51 ; p=0,001\right)$.

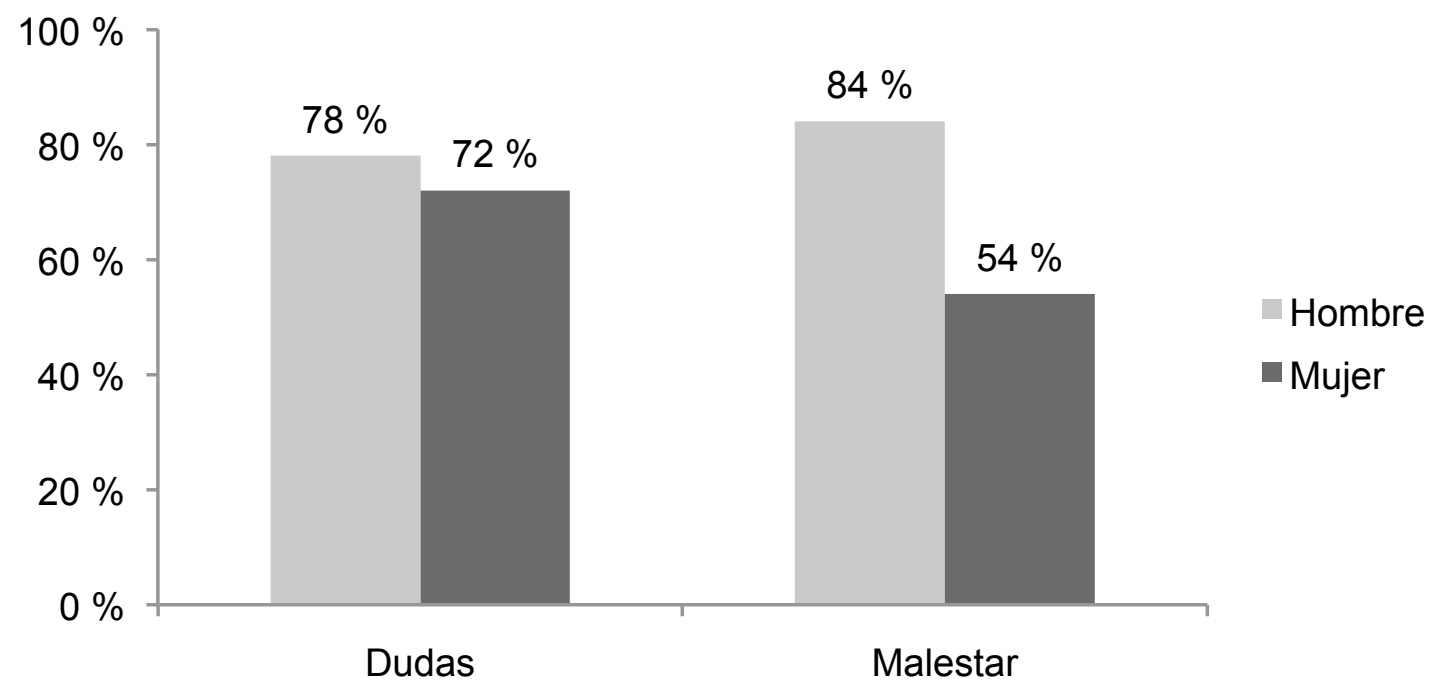

Figura 1. Porcentaje de hombres y mujeres con dudas y malestar con su propia orientación sexual.

En cuanto a la edad en la que definieron su actual orientación sexual, la media general fue a los 14,69 años (DT =3,83). Los hombres toman conciencia de su orientación sexual hacia los 14,32 años ( $D T=4,39$ ); en cambio, las mujeres tardan ligeramente un poco más, a los 15,06 años ( $D T=3,17$ ). No obstante, estas diferencias entre ambos grupos no resultaron significativas $(t=1,37 ; p=0,244)$.

\section{Conclusiones}

A la luz de los presentes resultados, tal y como se había observado en anteriores estudios (Soriano 2004), podemos confirmar que la vivencia de la homosexualidad puede llegar a ser un proceso complicado, ya que se observa una alta prevalencia de 
población homosexual que experimenta dudas y malestar con su orientación sexual. En cuanto a la edad de definición, alrededor de los 14-15 años es cuando, tanto chicos como chicas, toman conciencia de su orientación sexual.

En este sentido, los datos obtenidos apoyarían solamente la segunda hipótesis sobre la existencia de mayor porcentaje de hombres que hayan experimentado malestar con su orientación sexual, en comparación con las mujeres. En cuanto al resto de hipótesis, pese a existir mayor porcentaje de hombres que hayan experimentado dudas con su orientación sexual y ser los hombres quienes definen antes su actual orientación sexual, esas diferencias observadas con respecto a las mujeres no han resultado significativas.

Las altas prevalencias de dudas y malestar con la propia homosexualidad irían en consonancia con los resultados obtenidos en anteriores investigaciones (NebotGarcía, Giménez-García, Ruiz-Palomino, Iglesias-Campos y Ballester-Arnal 2018), en las que se había observado que la población no heterosexual mostraba más dudas y malestar con su orientación sexual respecto a las personas heterosexuales. Estos resultados podrían explicarse por el rechazo existente en la sociedad hacia la homosexualidad (Cornejo 2015; Parrott 2009; Parrott y Zeichner 2008; Poteat y Anderson 2012), siendo más notorio cuando se trata de hombres homosexuales (Petersen y Hyde 2011; Poteat y Anderson 2012), ya que la homosexualidad en los hombres es culturalmente percibida como una ruptura de los roles tradicionales de género (Parrott 2009; Parrott y Zeichner 2008), lo que podría explicar que este sector de la población exprese mayores niveles de malestar con su orientación sexual, en comparación con las mujeres homosexuales.

Por lo que respecta a la edad de definición, las diferencias no fueron significativas entre hombres y mujeres, pese a que en otros estudios se afirmaba que las mujeres solían darse cuenta de su deseo homosexual en edades más tardías, en comparación con los hombres (Peters en y Hyde 2011). Una posible explicación podría ser debido a las características de los participantes, como tener, mayoritariamente, estudios superiores.

Si bien este estudio ofrece información valiosa sobre la vivencia emocional de la población joven homosexual, no está exento de algunas limitaciones, como el tamaño muestral: quizás una mayor representación muestral facilitaría que los resultados fueran más concluyentes. Del mismo modo, tal y como se ha dicho anteriormente, sería conveniente equiparar a los participantes en cuanto al nivel de estudios, ya que la muestra estaba parcialmente sesgada hacia la población universitaria.

En cualquier caso, los hallazgos encontrados en este estudio hacen visible la necesidad de trabajar variables protectoras que faciliten una adecuada construcción de la identidad y de la orientación sexual, así como acompañar en el proceso de toma de conciencia y aceptación desde las instituciones sanitarias y educativas, con el fin de que la población homosexual tenga una vivencia más saludable de la propia identidad psicosexual.

\section{Referencias bibliográficas}

Agénor, Madina, Christina A. Muzny, Vanessa Schick, Erika L. Austin y Jennifer Potter. 2017. "Sexual orientation and sexual health services utilization among women in the United States». Preventive Medicine 95: 74-81.

Asociación Internacional de Lesbianas, Gays, Bisexuales, Trans e Intersex. 2017. Homofobia de Estado 2017. Estudio jurídico mundial sobre la orientación 
sexual en el derecho: criminalización, protección y reconocimiento. Ginebra: ILGA. Recuperado de:

http://ilga.org/downloads/2017/ILGA_Homofobia_de_Estado_2017_WEB.pdf

[Consultado el 10 de marzo de 2018].

Boletín Oficial de las Cortes Generales, 12 de mayo de 2017, núm. 122-1. Proposición de Ley contra la discriminación por orientación sexual, identidad o expresión de género y características sexuales, y de igualdad social de lesbianas, gais, bisexuales, transexuales, transgénero e intersexuales. Recuperado de: http://www.congreso.es/public_oficiales/L12/CONG/BO CG/B/BOCG-12-B-1221.PDF [Consultado el 3 de agosto de 2019].

Carnaghi, Andrea, Anne Maass y Fabio Fasoli. 2011. «Enhancing masculinity by slandering homosexuals: The role of homophobic epithets in heterosexual gender identity». Personality and Social Psychology Bulletin 37(12): 1655-1665.

Cornejo, Juan. 2015. «Componentes ideológicos de la homofobia». Límite. Revista de Filosofía y Psicología 7(26): 85-106.

Digoix, Marie, Marina Franchi, Jose Ignacio Pichardo Galán, Giulia Selmi, Matias de Stéfano Barbero, Matthias Thibeaud y Jose A. M. Vela. 2016. "Sexual orientation, family and kinship in France, Iceland, Italy and Spain». Families and Societies Working Paper 54(2016). Recuperado de:

http://www.familiesandsocieties.eu/wp-content/uploads/2016/08/WP54Digoixeta 12016.pdf [Consultado el 13 de mayo de 2018].

Glick, Peter, Candice Gangl, Samantha Gibb, Susan Klumpner y Emily Weinberg. 2007. "Defensive reactions to masculinity threat: More negative affect toward effeminate (but not masculine) gay men». Sex Roles 57(1-2): 55-59.

Kosciw, Joseph G., Neal A. Palmer y Ryan M. Kull. 2015. «Reflecting resiliency: openness about sexual orientation and/or gender identity and its relationship to well-being and educational outcomes for LGBT students». American Journal of Community Psychology 55(1-2): 167-178.

Ley $8 / 2017$, de 7 de abril, de la Generalitat, integral del reconocimiento del derecho a la identidad y a la expresión de género en la Comunidad Valenciana. Diari Oficial de la Generalitat Valenciana, núm. 8019, de 11 de abril de 2017, pp. 12310 a 12333 . Recuperado de:

https://www.dogv.gva.es/datos/2017/04/11/pdf/2017_3089.pdf [Consultado el 3 de agosto de 2019].

Ley $13 / 2005$, de 1 de julio, por la que se modifica el Código Civil en materia de derecho a contraer matrimonio. Boletín Oficial del Estado, núm. 157, de 2 de julio de 2005, pp. 23632 a 23634. Recuperado de:

https://www.boe.es/boe/dias/2005/07/02/pdfs/A23632-23634.pdf [Consultado el 3 de agosto de 2019].

Martxueta, Aitor y Juan Etxeberria. 2014. "Análisis diferencial retrospectivo de las variables de salud mental en lesbianas, gais y bisexuales (LGB) víctimas de bullying homofóbico en la escuela». Revista de Psicopatología y Psicología Clínica 19(1): 23-35.

Nebot-García, Juan Enrique, Cristina Giménez-García, Estefanía Ruiz-Palomino, Paula Iglesias-Campos y Rafael Ballester-Arnal. 2018. «Malestar emocional asociado a la orientación sexual en personas mayores». International Journal of Developmental and Educational Psychology: INFAD. Revista de Psicología 1(4): 49-56.

Newcomb, Michael E. y Brian Mustanski. 2010. «Internalized homophobia and internalizing mental health problems: A meta-analytic review». Clinical Psychology Review 30(8): 1019-1029.

Parrott, Dominic J. 2009. «Aggression toward gay men as gender role enforcement: Effects of male role norms, sexual prejudice, and masculine gender role stress». Journal of Personality 77(4): 1137-1166. 
Parrott, Dominic J. y Amos Zeichner. 2008. «Determinants of anger and physical aggression based on sexual orientation: An experimental examination of hypermasculinity and exposure to male gender role violations». Archives of Sexual Behavior 37(6): 891-901.

Petersen, Jennifer L. y Janet Shibley Hyde. 2011. "Gender differences in sexual attitudes and behaviors: A review of meta-analytic results and large datasets». Journal of Sex Research 48(2-3): 149-165.

Poteat, V. Paul y Carolyn J. Anderson. 2012. "Developmental changes in sexual prejudice from early to late adolescence: The effects of gender, race, and ideology on different patterns of change». Developmental Psychology 48(5): 1403-1415.

Soriano, Sonia. 2004. Cómo se vive la homosexualidad y el lesbianismo (2. ${ }^{a}$ edición). Salamanca: Amarú. 